\title{
Clinical outcomes of difficult-to-wean patients with ventilator dependency at intensive care unit discharge
}

\author{
Jung Mo Lee', Sun-Min Lee', Joo Han Song ${ }^{2}$, Young Sam Kim² \\ ${ }^{1}$ Division of Pulmonology, Department of Internal Medicine, National Health Insurance Service Ilsan Hospital, Goyang: ${ }^{2}$ Division of Pulmonary and Critical \\ Care Medicine, Department of Internal Medicine, Institute of Chest Diseases, Severance Hospital, Yonsei University College of Medicine, Seoul, Korea
}

Background: Ventilator-dependent patients in the intensive care unit (ICU) who are difficult to wean from invasive mechanical ventilation (IMV) have been increasing in number. However, data on the clinical outcomes of difficult-to-wean patients are lacking. We aimed to evaluate clinical outcomes in patients discharged from the ICU with tracheostomy and ventilator dependency.

Methods: We retrospectively investigated clinical course and survival in patients requiring home mechanical ventilation (HMV) with a tracheostomy and difficulty weaning from IMV during medical ICU admission from September 2013 through August 2016 at Severance Hospital, Yonsei University, Seoul, Korea.

Results: Of 84 difficult-to-wean patients who were started on HMV in the medical ICU, 72 survived, were discharged from the ICU, and were included in this analysis. HMV was initiated after a median of 23 days of IMV, and the successful weaning rate was $46 \%(n=33)$. In-hospital mortality rate was significantly lower in the successfully weaned group than the unsuccessfully weaned group ( $0 \%$ vs. $23.1 \%$, respectively; $P=0.010)$. Weaning rates were similar according to primary diagnosis, but high body mass index (BMI), low Acute Physiologic Assessment and Chronic Health Evaluation (APACHE) II score at ICU admission, and absence of neuromuscular disease were associated with weaning success. After a median follow-up of 4.6 months (range, 1-27 months) for survivors, 3-month $(n=64)$ and 6-month $(n=59)$ survival rates were $82.5 \%$ and $72.2 \%$, respectively. Survival rates were higher in the successfully weaned group than the unsuccessfully weaned group at 3 months $(96.4 \%$ vs. $69.0 \% ; P=0.017)$ and 6 months (84.0\% vs. $62.1 \%$; $P=0.136$ ) following ICU discharge.

Conclusions: In summary, $46 \%$ of patients who started HMV were successfully weaned from the ventilator in general wards. High BMI, low APACHE II score, and absence of neuromuscular disease were factors associated with weaning success.

Key Words: intensive care unit; mechanical ventilators; ventilator weaning

\section{INTRODUCTION}

Advances in intensive care medicine have increased the number of patients surviving episodes of acute respiratory failure or chronic respiratory failure. At the same time, however, there has also been an increase in the number of patients who become partially or entirely dependent on mechanical ventilation or other intensive care treatments [1]. Thus, prolonged dependence on mechanical ventilation after critical illness is an emerging public health prob-

\section{Original Article}

Received: April 22, 2020

Revised: July 3, 2020

Accepted: July 22, 2020

Corresponding author Young Sam Kim

Division of Pulmonology, Department of Internal Medicine, Severance Hospital, Institute of Chest Diseases, Yonsei University College of Medicine, 50-1 Yonsei-ro, Seodaemun-gu, Seoul 03722, Korea Tel: +82-2-2228-1971 Fax: +82-2-393-6884 E-mail: ysamkim@yuhs.ac

Copyright (๑) 2020 The Korean Society of Critical Care Medicine

This is an Open Access article distributed under the terms of Creative Attributions Non-Commercial License (https:// creativecommons.org/li-censes/by-nc/4.0/) which permits unrestricted noncommercial use, distribution, and reproduction in any medium, provided the original work is properly cited. 
lem. Up to $40 \%$ of patients entering the intensive care unit (ICU) require mechanical ventilation, and nearly $20 \%$ of these patients are expected to have increased ICU stay due to difficult weaning [2]. In the United States, there are about 100,000 such patients, and the cost exceeds US $\$ 20$ billion per year. This cost is predicted to be greater than US $\$ 50$ billion by 2020 [1].

In difficult-to-wean patients, weaning success is defined as extubation followed by absence of ventilatory support for 48 hours or 7-14 days without the need for mechanical ventilation. The success rate of weaning is about $30 \%-53 \%$, and the average time to liberation is 16 to 37 days. Moreover, failure to wean from mechanical ventilation within 60 days is likely to lead to persistent failure [3-5]. Some countries operate respiratory intensive care units (RICUs) or weaning centers (WCs), and such facilities show good success rates and long-term survival $[3,6,7]$. There is currently no such facility in Korea, so most difficult-to-wean patients remain in the ICU or are transferred to a general ward with application of home mechanical ventilation (HMV). Although some studies of patients with prolonged weaning in an ICU have been performed, no prior studies have investigated weaning outcomes of patients transferred to the general ward with HMV [8-12]. Therefore, in this study, we analyzed the clinical outcomes of patients with ventilator dependency upon discharge from the ICU and identified prognostic factors that predicted weaning success in such patients.

\section{MATERIALS AND METHODS}

\section{Research Ethics}

This study was conducted in accordance with the amended Declaration of Helsinki. The study protocol was reviewed and approved by the Institutional Review Board of Yonsei University Health System, Severance Hospital, Seoul, Korea (IRB No. 4-2017-0681), and by the National Health Insurance Service Ilsan Hospital, Gyeonggi-do, Korea (IRB No. 2018-04-033).

\section{Subjects}

We retrospectively reviewed the medical records of patients requiring a HMV with a tracheostomy due to difficult weaning from invasive mechanical ventilation (IMV). Patients who were discharged from the medical ICU to a general ward with ventilator support with HMV between September 2013 and August 2016 at Severance Hospital, Yonsei University in Korea were included. Severance Hospital has a 30-bed medical ICU within a 2,182-bed tertiary medical center containing 260 ICU beds. Bed operation rate of the total ICU was $80.7 \%$ at the time

\section{KEY MESSAGES}

- We retrospectively investigated clinical course and survival in patients requiring home mechanical ventilation (HMV).

- A total of $46 \%$ of patients who started HMV in the medical intensive care unit were successfully weaned from the ventilator.

- High body mass index, low Acute Physiologic Assessment and Chronic Health Evaluation II score, and absence of neuromuscular disease were factors associated with weaning success.

of this study. We defined difficult-to-wean patients as cases in which a tracheostomy was performed after at least 10 days of mechanical ventilation [13]. Successful weaning was defined as at least 7 days of complete ventilator independence [14]. Patients who died before successful weaning were considered weaning failures. We followed the guidelines specified for neuromuscular blockade and sedative agents, lung-protective ventilation, and spontaneous breathing trials [15-18]. All patients requiring IMV were screened for weaning readiness using a standardized weaning protocol (Supplementary Figure 1). Patients underwent a spontaneous breathing trial (SBT) when their clinical condition was considered stable. The SBT consisted of breathing with a T-piece at 9-10 L/min with a $40 \%$ inspiratory oxygen fraction and lasted 30-120 minutes. If this trial was successful, extubation proceeded. If a patient failed this trial, IMV was restarted, and the patient was reassessed for weaning and SBT the next day. The need for tracheostomy was considered for patients with mechanical ventilator dependency of 7 days or more. HMV application was recommended for patients for whom weaning was unsuccessful after two or more SBT attempts.

\section{Data Collection}

Clinical and demographic characteristics of age, sex, comorbid conditions, causes of ICU admission, albumin and hemoglobin levels at the start of HMV, durations of IMV and HMV, lengths of stay in the ICU and the hospital, in-hospital mortality, 3- and 6-month survival rates, and discharge type were recorded. Acute Physiologic Assessment and Chronic Health Evaluation (APACHE) II score, Charlson comorbidity index, body mass index (BMI), and European Cooperative Oncology Group (ECOG) score were determined at the time of ICU admission [19]. 


\section{Statistical Analysis}

Continuous variables were compared between groups using the Mann-Whitney U-test. Categorical variables are presented as numbers or percentages and were compared using the Chisquare or Fisher's exact test. To determine factors predictive of weaning success, logistic regression analysis with stepwise and backward selection procedures was performed. Variables with $\mathrm{P}<0.2$ were included in multiple logistic regression, and any predictor in which the $\mathrm{P}$-value in the multivariate model exceeded 0.05 was eliminated. All adjusted P-values $<0.05$ were considered statistically significant. All statistical analyses were carried out using IBM SPSS version 23.0 (IBM Corp., Armonk, NY, USA).

\section{RESULTS}

During the study period, 1,217 patients required IMV for at least 24 hours in the medical ICU, and 199 patients were difficult-to-wean and needed tracheostomy after at least 10 days of mechanical ventilation [13]. Eighty-four of these patients started HMV for difficult weaning from IMV and were included in this study. Of these, 12 patients died while in the ICU. The remaining 72 individuals were discharged from the ICU to a general ward when the following criteria were met: (1) hemodynamic stability off inotropes, (2) absence of sepsis, (3) ventilation via tracheostomy, and (4) ability to cooperate with therapy (no continuous sedation). Of these 72 patients, 33 (46\%) were weaned from HMV successfully (Figure 1). The baseline characteristics of patients requiring HMV according to weaning success or failure are shown in Table 1. Men were predominant in both groups. The median ages of the groups did not differ ( 64.0 vs. 64.0 years, $\mathrm{P}=0.799$ ). There was no significant difference in Charlson comorbidity index between the two groups. The prevalence of diabetes mellitus, renal insufficiency, and malignancy was not different between the two groups. However, neurologic disease was more frequent in the weaning failure group than the weaning success group (61.5\% vs. $30.3 \%, \mathrm{P}=0.016)$.

Primary causes of ICU admission were not different between the two groups, and the most common cause was a respiratory condition. APACHE II score on ICU admission was higher in the weaning failure group than in the weaning success group (23.6 vs. 19.5, $\mathrm{P}=0.013$ ), while $\mathrm{BMI}$ was higher in the weaning success group (21.1 vs. $23.7, \mathrm{P}=0.036$ ). Most patients had an ECOG score of 4 , and some had 3 points, but there was no difference in mean ECOG scores between the two groups. There was no significant difference in albumin hemoglobin level at the onset of HMV. Pulmonary rehabilitation was performed in 15 patients and was more frequent in the weaning success group than the weaning failure group, but this difference did not reach statistical significance $(30.3 \%$ vs. $12.8 \%, \mathrm{P}=0.126)$.

Clinical outcomes for patients are shown in Table 2 and Figure 2. Of the patients in the weaning success group, 17 (52\%) were discharged home, and 15 were still alive after 3 months. Among 16 patients (48\%) discharged to other facilities, 12 were alive after 3 months. Nine of the patients in the failure group died at the hospital without discharge. Five patients (13\%) were discharged home, and 22 patients (56\%) were discharged to other facilities, with 17 patients surviving after 3 months.

Duration from IMV to HMV initiation or duration from HMV to ICU discharge did not differ between the two groups (21.0 vs. 23.0 days, $\mathrm{P}=0.941$ and 4.0 vs. 3.0 days; $\mathrm{P}=0.177$, respectively). Duration of HMV maintenance was longer in the weaning failure group than the weaning success group, but this difference was not statistically significant (39.0 vs. 31.0 days, respectively; $\mathrm{P}=0.331$ ). There was also no significant difference

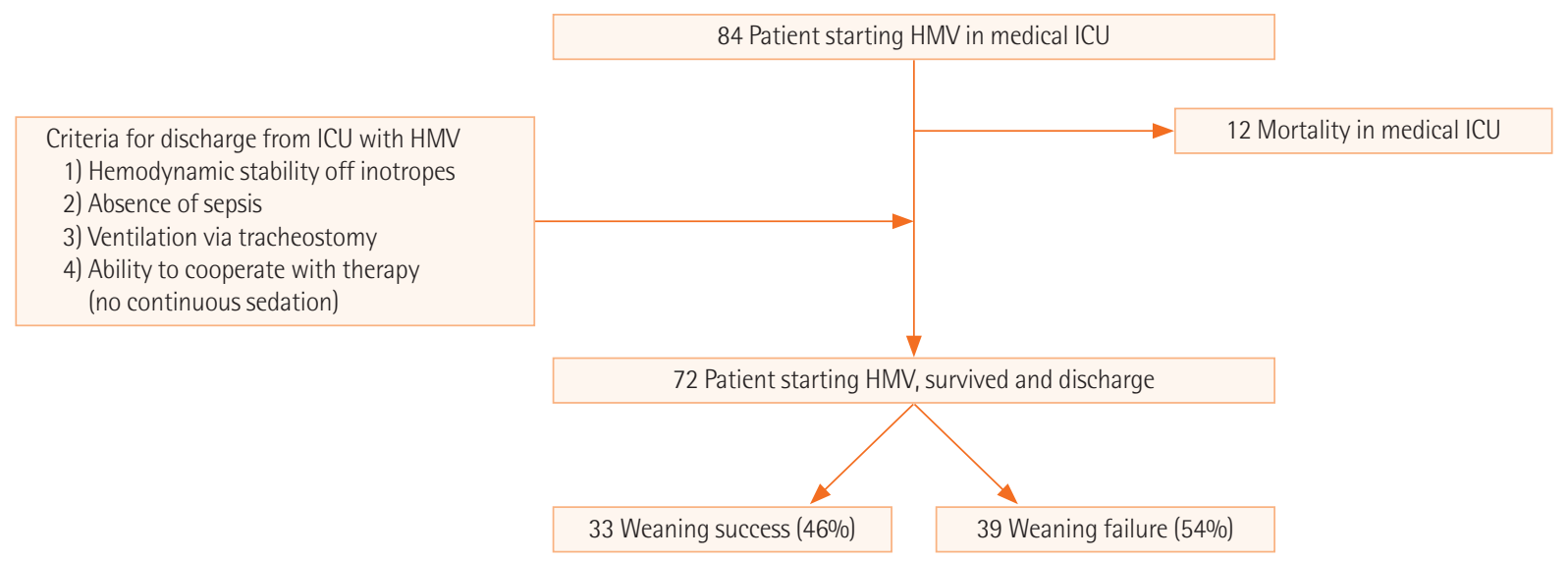

Figure 1. Flowchart of patient enrollment. HMV: home mechanical ventilation; ICU: intensive care unit. 
Table 1. Baseline characteristics of patients requiring HMV

\begin{tabular}{|c|c|c|c|c|}
\hline Baseline characteristics & Total $(n=72)$ & Weaning failure $(n=39)$ & Weaning success $(n=33)$ & P-value \\
\hline Sex & & & & 0.983 \\
\hline Male & $47(65.3)$ & $26(66.7)$ & $21(63.6)$ & \\
\hline Female & $25(34.7)$ & $13(33.3)$ & $12(36.4)$ & \\
\hline Age (yr) & $64.0(50.5-72.5)$ & $64.0(52.5-67.5)$ & $64.0(45.0-74.0)$ & 0.799 \\
\hline Charlson comorbidity index & $1.0(0.0-3.5)$ & $1.0(0.0-3.0)$ & $2.0(0.0-4.0)$ & 0.520 \\
\hline \multicolumn{5}{|l|}{ Comorbid condition } \\
\hline Diabetes mellitus & $21(29.2)$ & $11(28.2)$ & $10(30.3)$ & 1.000 \\
\hline Renal insufficiency & $27(37.5)$ & $13(33.3)$ & $14(42.4)$ & 0.583 \\
\hline Malignancy & $20(27.8)$ & $10(25.6)$ & $10(30.3)$ & 0.860 \\
\hline Neurologic disease & $34(47.2)$ & $24(61.5)$ & $10(30.3)$ & 0.016 \\
\hline Cause of ICU admission & & & & 0.738 \\
\hline Respiratory & $58(80.6)$ & $31(79.5)$ & $27(81.8)$ & \\
\hline Cardiovascular & $7(9.7)$ & $3(7.7)$ & $4(12.1)$ & \\
\hline Sepsis other than pneumonia & $4(5.6)$ & $3(7.7)$ & $1(3.0)$ & \\
\hline Neurologic & $3(4.2)$ & $2(5.1)$ & $1(3.0)$ & \\
\hline APACHE II score (at ICU admission) & $22.0(16.0-27.0)$ & $24.0(17.5-28.5)$ & $20.0(16.0-24.0)$ & 0.022 \\
\hline Albumin on HMV & $2.6(2.3-2.8)$ & $2.5(2.2-2.8)$ & $2.7(2.4-2.8)$ & 0.250 \\
\hline Hemoglobin on HMV & $9.3(8.7-10.4)$ & $9.1(8.3-10.2)$ & $9.7(9.0-10.4)$ & 0.099 \\
\hline Pulmonary rehabilitation & $15(20.8)$ & $5(12.8)$ & $10(30.3)$ & 0.126 \\
\hline
\end{tabular}

Values are presented as number (\%) or median (interquartile range).

HMV: home mechanical ventilation; ICU: intensive care unit; APACHE: Acute Physiologic Assessment and Chronic Health Evaluation.

Table 2. Clinical outcomes of patients requiring HMV

\begin{tabular}{lcccc}
\hline Clinical outcome & $\begin{array}{c}\text { Weaning failure } \\
(\mathrm{n}=39)\end{array}$ & $\begin{array}{c}\text { Weaning success } \\
(\mathrm{n}=33)\end{array}$ & $\begin{array}{c}\text { Total } \\
(\mathrm{n}=72)\end{array}$ & P-value \\
\hline Duration from IMV to HMV initiation (day) & $21.0(10.5-41.0)$ & $23.0(15.0-35.0)$ & $22.5(12.0-38.5)$ & 0.941 \\
Duration from HMV to ICU discharge (day) & $4.0(2.0-7.0)$ & $3.0(2.0-6.0)$ & $3.0(2.0-6.0)$ & 0.177 \\
Duration of HMV maintenance (day) & $39.0(14.5-97.0)$ & $31.0(21.0-49.0)$ & $36.5(16.0-62.5)$ & 0.331 \\
Duration from pulmonary rehabilitation to HMV weaning (day) & & $40.5(0.0-54.0)$ & & \\
Length of stay in ICU & $26.0(13.5-57.5)$ & $26.0(17.0-36.0)$ & $26.0(14.0-54.5)$ & 0.747 \\
Length of stay in hospital & $80.0(36.0-158.5)$ & $116.0(79.0-167.0)$ & $105.5(61.5-165.0)$ & 0.094 \\
In-hospital mortality rate & $9(23.1)$ & 0 & $9(12.5)$ & 0.010 \\
3-Month survival rate & $20(69.0)$ & $27(96.4)$ & $47(82.5)$ & 0.017 \\
6-Month survival rate & $18(62.1)$ & $21(84.0)$ & $39(72.2)$ & 0.136 \\
\hline
\end{tabular}

Values are presented as median (interquartile range) or number (\%).

HMV: home mechanical ventilation; IMV: invasive mechanical ventilation; ICU: intensive care unit.

between groups in ICU length of stay or hospital length of stay (26.0 vs. 26.0 days, $\mathrm{P}=0.747$ and 80.0 vs. 116.0 days, $\mathrm{P}=0.094$ ).

Unlike other outcomes, the in-hospital mortality rate was higher and the 3-month survival rate was lower in the weaning failure group than the weaning success group $(23.1 \%$ vs. $0.0 \%, \mathrm{P}=0.010$ and $69.0 \%$ vs. $96.4 \%, \mathrm{P}=0.017$ ). The 6 -month survival rate was higher in the weaning success group than the weaning failure group, but the difference between groups was not significant $(84.0 \%$ vs. $62.1 \%, \mathrm{P}=0.136)$.

Table 3 shows the results of univariate and multivariate analyses to identify predictive factors associated with weaning success in patients requiring HMV. In the univariate analysis, three clinical characteristics were associated with weaning success (all $\mathrm{P}<0.05$ ): neurologic disease as a comorbid condition, APA- 


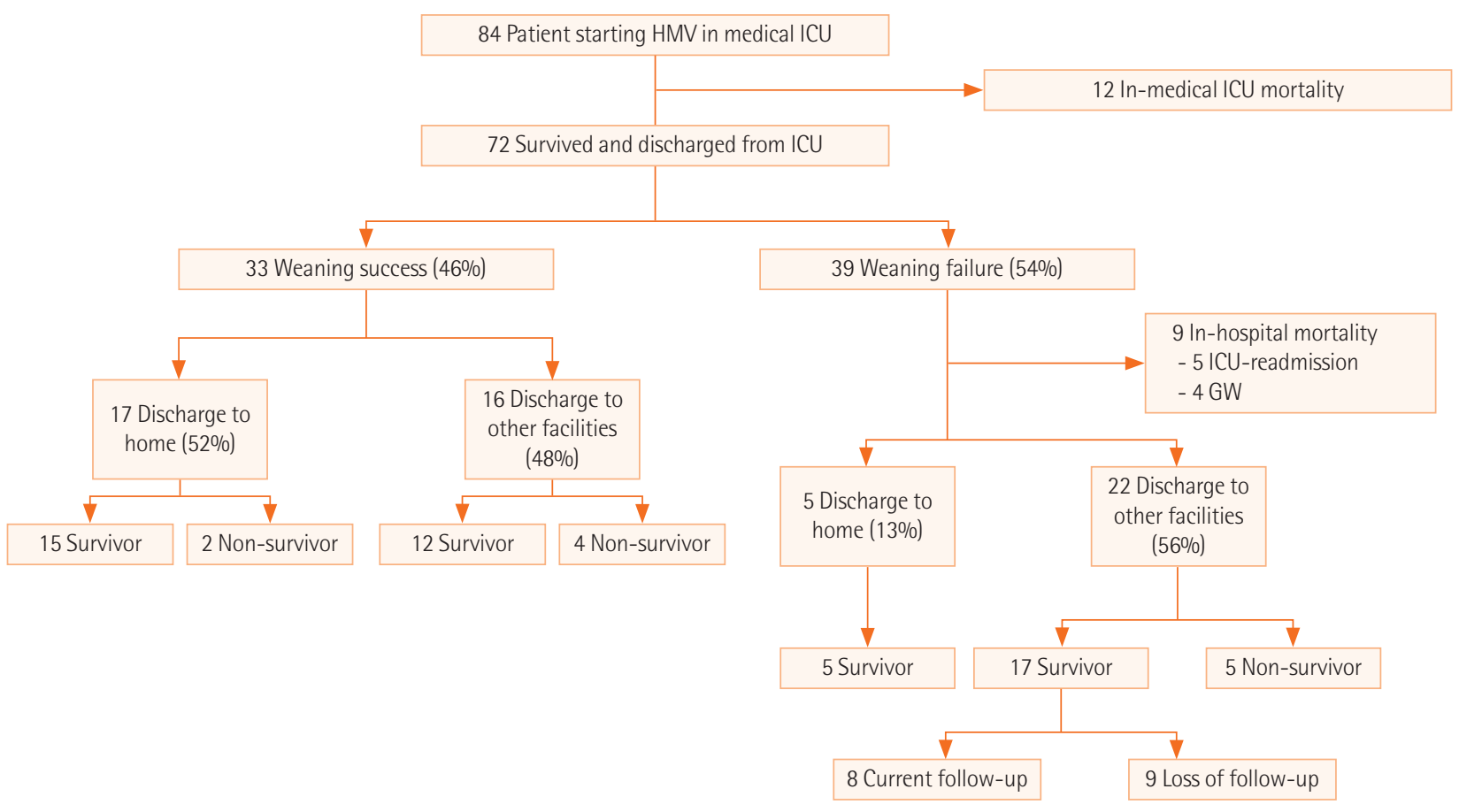

Figure 2. Flow diagram of patient outcomes with home mechanical ventilation (HMV). ICU: intensive care unit.

Table 3. Factors predictive of weaning success

\begin{tabular}{|c|c|c|c|c|}
\hline \multirow{2}{*}{ Variable } & \multicolumn{2}{|c|}{ Univariate analysis } & \multicolumn{2}{|c|}{ Multivariate analysis } \\
\hline & OR $(95 \% \mathrm{Cl})$ & P-value & OR $(95 \% \mathrm{Cl})$ & P-value \\
\hline Neurologic disease & $0.27(0.10-0.71)$ & 0.009 & - & - \\
\hline Cerebrovascular disease & $0.76(0.18-2.92)$ & 0.691 & - & - \\
\hline Neuromuscular disease & $0.26(0.08-0.74)$ & 0.015 & $0.26(0.08-0.78)$ & 0.020 \\
\hline APACHE II score (ICU admission) & $0.91(0.84-0.98)$ & 0.017 & $0.92(0.84-0.99)$ & 0.038 \\
\hline Albumin level (ICU discharge) & $3.17(1.03-11.35)$ & 0.057 & - & - \\
\hline Hemoglobin level (ICU discharge) & $1.37(0.93-2.10)$ & 0.126 & - & - \\
\hline Pulmonary rehabilitation & $2.96(0.92-10.56)$ & 0.076 & - & - \\
\hline
\end{tabular}

OR: odds ratio; Cl: confidence interval; APACHE: Acute Physiologic Assessment and Chronic Health Evaluation; ICU: intensive care unit.

CHE II score, and BMI on ICU admission. When neurologic disease was classified as cerebrovascular or neuromuscular disease, only neuromuscular disease was associated with weaning success $(\mathrm{P}=0.015)$. In the multivariate analysis, neuromuscular disease as a comorbid condition (odds ratio [OR], 0.23; 95\% confidence interval [CI], 0.06-0.72; $\mathrm{P}=0.015)$ and APACHE II score on ICU admission (OR, 0.92; 95\% CI, 0.84-0.99; $\mathrm{P}=0.040)$ were associated with a significantly decreased weaning success rate in patients on HMV. In contrast, BMI on ICU admission (OR, 1.16; 95\% CI, 1.03-1.36; $\mathrm{P}=0.031$ ) was associated with a significantly increased weaning success rate in patients on HMV. An ROC curve of APACHEII score on ICU admission for predicting weaning success in patients on HMV is presented in Supplementary Figure 2.

\section{DISCUSSION}

We investigated the clinical outcomes of difficult-to-wean patients who required HMV at ICU discharge and prognostic factors predictive of weaning success in these patients. In our study cohort, the rate of successful weaning of HMV patients was $46 \%$. APACHE II score at ICU admission was lower and BMI was higher in the weaning success group than the weaning failure group. In-hospital mortality rate and 3-month and 6-month mortality rates were better for the successfully weaned group than the unsuccessfully weaned group. Additionally, 
multivariate analysis demonstrated that higher BMI, lower APACHE II score, and absence of neuromuscular disease were statistically significant predictors of weaning success.

This is the first study in Korea to assess the clinical outcomes of difficult-to-wean patients in a non-ICU setting. At our institute, we perform tracheostomy and care for difficult-to-wean patients in general wards. Some countries operate RICUs or WCs, and such facilities show a high success rate and good long-term survival $[3,6,7]$. There is currently no such facility in Korea, so most difficult-to-wean patients stay in the ICU. Our results indicate that transferring these patients to a general ward and weaning them from HMV is a viable alternative approach to RICUs or WCs.

The rate of successful liberation from a ventilator in critically ill patients treated with prolonged mechanical ventilation varies significantly from study to study. Damuth et al. [20] reported that the pooled proportion of patients liberated from mechanical ventilation was $50 \%(47 \%-53 \%)$ in a meta-analysis of studies on prolonged mechanical ventilation. In our research, the successful weaning rate of HMV patients was $46 \%$. Differences in patient composition such as race, country, and hospital characteristics may be responsible for these differences between studies, making it difficult to compare results between studies and limiting the generalizability of individual study findings.

In our institution, all patients in the ICU with ventilator dependency are screened daily for weaning readiness by a standardized weaning protocol. The need for tracheostomy is considered for patients with a mechanical ventilator dependency of 7 days or more. HMV application is recommended for patients who fail to wean after two or more SBT attempts. In this study, there was no difference in length of stay of ICU and duration from IMV to HMV initiation between the HMV weaning success and failure groups. However, the length of stay in the hospital was 80 days in the weaning failure group, which was shorter than that in the success group. This is likely due to the small proportion of patients discharged home and the high rate of discharge to other facilities in the weaning failure group. Unlike the ICU, discharge from the ward to a home or another medical facility was not enforced according to an established protocol. The absence of discharge criteria is a limitation of this study.

In our study, factors associated with weaning failure in patients with HMV were presence of neurologic disease, higher APACHE II score, and lower BMI. This is in contrast to other studies, where cause of ICU admission, serum hemoglobin, and albumin level were found to be associated with weaning failure $[8,21,22]$. Several studies have shown that higher BMI is associated with lower mortality in critically ill patients. This is likely because a low BMI is related to low protein and energy intake, malnutrition, and inadequate nutritional reserve, resulting in the inability to compensate for the stress of critical illness [23-25]. These characteristics may explain the association between higher BMI and weaning success rate in our study. Furthermore, some of the patients included in the current study underwent systematic respiratory rehabilitation. The weaning success rate of these patients was $58.8 \%$, much higher than the rates reported in other studies. Pulmonary rehabilitation includes the daily physical therapy of inspiratory muscle strength training. The absence of neuromuscular disease and intact muscle strength are essential for weaning, indicating that respiratory rehabilitation is necessary for difficult-to-wean patients. Additional studies are needed to determine the exact effects of pulmonary rehabilitation.

There are several limitations to this study. First, our study was conducted at a single, university-affiliated, tertiary referral hospital and included only difficult-to-wean patients who had undergone tracheostomy and HMV in general wards. This may limit the generalizability of our findings to other difficultto-wean patients or other centers. Secondly, unlike the ICU, discharge from the ward to home or another medical facility was not enforced according to an established protocol. In addition, although patients who were transferred to other institutions were included and could be followed-up for a short time, it was difficult to track these patients for more than 6 months. Further studies are needed to determine survival rates at greater than one year. Furthermore, because our research was retrospective, we were not able to examine various other factors that could affect weaning failure.

In summary, $46 \%$ of patients on HMV were successfully weaned from the ventilator in general wards. High BMI, low APACHE II score, and presence of neuromuscular disease were factors associated with weaning success.

\section{CONFLICT OF INTEREST}

No potential conflict of interest relevant to this article was reported.

\section{ACKNOWLEDGMENTS}

This work was supported by a National Health Insurance Service Ilsan Hospital grant (NHIMC2018CR033).

We submitted the abstract of this study in ESICM LIVES 
2018 (Paris, France. 20-24 October 2018).

\section{ORCID}

Jung Mo Lee

https://orcid.org/0000-0002-9972-8506

Sun-Min Lee

https://orcid.org/0000-0003-3325-3242

Young Sam Kim

https://orcid.org/0000-0001-9656-8482

\section{AUTHOR CONTRIBUTIONS}

Conceptualization: YSK, SL. Data curation: JHS, JML. Formal analysis: JHS, JML. Funding acquisition: JML. Methodology: YSK, JHS. Project administration: JML. Visualization: JHS, JML. Writing-original draft: JML. Writing-review \& editing: YSK, SL.

\section{SUPPLEMENTARY MATERIALS}

Supplementary materials can be found at https://doi.org/10. 4266/acc.2020.00199.

\section{REFERENCES}

1. Ambrosino N, Gabbrielli L. The difficult-to-wean patient. Expert Rev Respir Med 2010;4:685-92.

2. Esteban A, Anzueto A, Alía I, Gordo F, Apezteguía C, Pálizas F, et al. How is mechanical ventilation employed in the intensive care unit? An international utilization review. Am J Respir Crit Care Med 2000;161:1450-8.

3. Boles JM, Bion J, Connors A, Herridge M, Marsh B, Melot C, et al. Weaning from mechanical ventilation. Eur Respir J 2007; 29:1033-56.

4. MacIntyre NR, Cook DJ, Ely EW Jr, Epstein SK, Fink JB, Heffner JE, et al. Evidence-based guidelines for weaning and discontinuing ventilatory support: a collective task force facilitated by the American College of Chest Physicians; the American Association for Respiratory Care; and the American College of Critical Care Medicine. Chest 2001;120(6 Suppl):375S395S.

5. Kahn JM, Carson SS, Angus DC, Linde-Zwirble WT, Iwashyna TJ. Development and validation of an algorithm for identifying prolonged mechanical ventilation in administrative data. Health Serv Outcomes Res Methodol 2009;9:117-32.

6. Carpenè N, Vagheggini G, Panait E, Gabbrielli L, Ambrosino N. A proposal of a new model for long-term weaning: respiratory intensive care unit and weaning center. Respir Med 2010; 104:1505-11.

7. Bigatello LM, Stelfox HT, Berra L, Schmidt U, Gettings EM.
Outcome of patients undergoing prolonged mechanical ventilation after critical illness. Crit Care Med 2007;35:2491-7.

8. Shin HJ, Chang JS, Ahn S, Kim TO, Park CK, Lim JH, et al. Clinical factors associated with weaning failure in patients requiring prolonged mechanical ventilation. J Thorac Dis 2017;9: 143-50.

9. Lee SH, Kim MJ, Jeong ES, Jo EJ, Eom JS, Mok JH, et al. Outcomes and prognostic factors in patients with prolonged acute mechanical ventilation: a single-center study in Korea. J Crit Care 2015;30:1016-20.

10. Lee K, Hong SB, Lim CM, Koh Y. Sequential organ failure assessment score and comorbidity: valuable prognostic indicators in chronically critically ill patients. Anaesth Intensive Care 2008;36:528-34.

11. Kim MH, Cho WH, Lee K, Kim KU, Jeon DS, Park HK, et al. Prognostic factors of patients requiring prolonged mechanical ventilation in a medical intensive care unit of Korea. Tuberc Respir Dis (Seoul) 2012;73:224-30.

12. Mok JH, Kim YH, Jeong ES, Eom JS, Kim MH, Kim KU, et al. Clinical application of the ProVent score in Korean patients requiring prolonged mechanical ventilation: a 10-year experience in a university-affiliated tertiary hospital. J Crit Care 2016;33:158-62.

13. Nelson JE, Cox CE, Hope AA, Carson SS. Chronic critical illness. Am J Respir Crit Care Med 2010;182:446-54.

14. Scheinhorn DJ, Chao DC, Stearn-Hassenpflug M, LaBree LD, Heltsley DJ. Post-ICU mechanical ventilation: treatment of 1,123 patients at a regional weaning center. Chest 1997;111: 1654-9.

15. Slomka J, Hoffman-Hogg L, Mion LC, Bair N, Bobek MB, Arroliga AC. Influence of clinicians' values and perceptions on use of clinical practice guidelines for sedation and neuromuscular blockade in patients receiving mechanical ventilation. Am J Crit Care 2000;9:412-8.

16. dos Santos CC, Slutsky AS. Protective ventilation of patients with acute respiratory distress syndrome. Crit Care 2004;8: 145-7.

17. Gillette MA, Hess DR. Ventilator-induced lung injury and the evolution of lung-protective strategies in acute respiratory distress syndrome. Respir Care 2001;46:130-48.

18. Epstein SK. Decision to extubate. Intensive Care Med 2002;28: 535-46.

19. Charlson ME, Pompei P, Ales KL, MacKenzie CR. A new method of classifying prognostic comorbidity in longitudinal studies: development and validation. J Chronic Dis 1987;40:37383.

20. Damuth E, Mitchell JA, Bartock JL, Roberts BW, Trzeciak S. 
Long-term survival of critically ill patients treated with prolonged mechanical ventilation: a systematic review and metaanalysis. Lancet Respir Med 2015;3:544-53.

21. Lai YC, Ruan SY, Huang CT, Kuo PH, Yu CJ. Hemoglobin levels and weaning outcome of mechanical ventilation in difficult-to-wean patients: a retrospective cohort study. PLoS One 2013;8:e73743.

22. Clochesy JM, Daly BJ, Montenegro HD. Weaning chronically critically ill adults from mechanical ventilatory support: a descriptive study. Am J Crit Care 1995;4:93-9.

23. Lim SY, Choi WI, Jeon K, Guallar E, Koh Y, Lim CM, et al. Body mass index and mortality in Korean intensive care units: a prospective multicenter cohort study. PLoS One 2014;9:e90039.

24. Kim H, Kim J, Seo C, Lee M, Cha MU, Jung SY, et al. Body mass index is inversely associated with mortality in patients with acute kidney injury undergoing continuous renal replacement therapy. Kidney Res Clin Pract 2017;36:39-47.

25. Pickkers P, de Keizer N, Dusseljee J, Weerheijm D, van der Hoeven JG, Peek N. Body mass index is associated with hospital mortality in critically ill patients: an observational cohort study. Crit Care Med 2013;41:1878-83. 


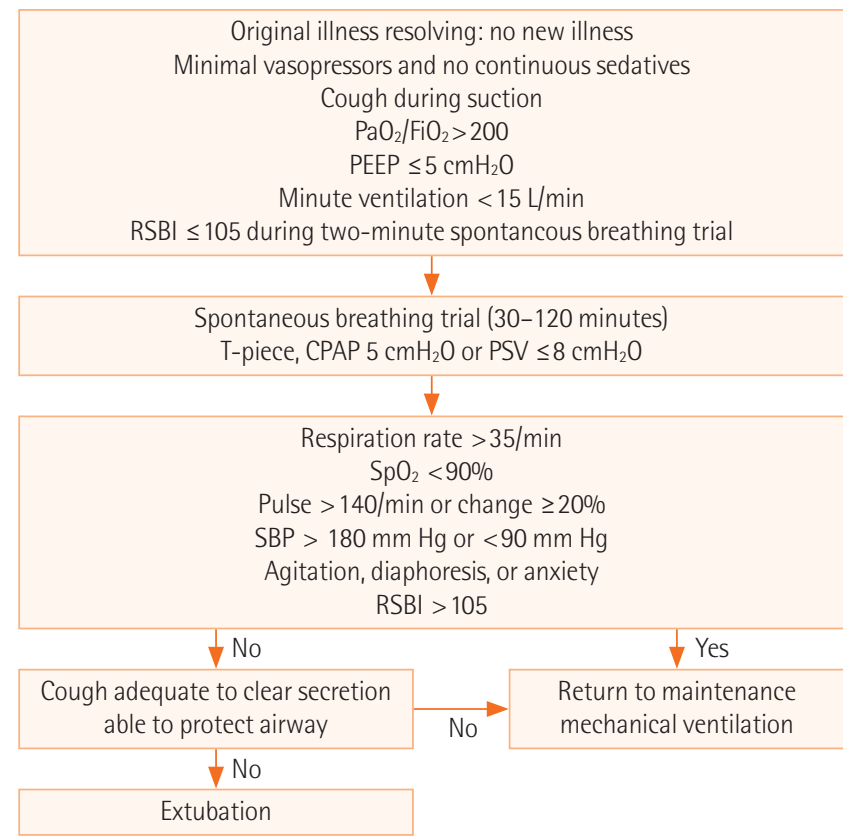

Supplementary Figure 1. Ventilator weaning protocol applied in this study. PEEP: positive end-expiratory pressure; RSBI: rapid shallow breathing index; CPAP: continuous positive airway pressure; PSV: pressure support ventilation; SBP: systolic blood pressure.

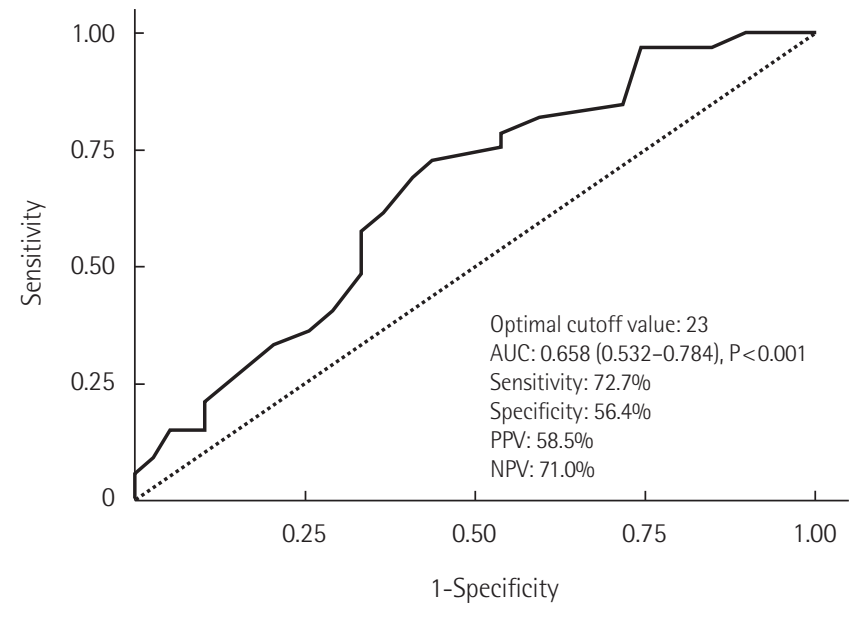

Supplementary Figure 2. Receiver operating characteristic curve of Acute Physiologic Assessment and Chronic Health Evaluation II score on intensive care unit admission for predicting weaning success in patients requiring home mechanical ventilation. AUC: area under the curve; PPV: positive predictive value; NPV: negative predictive value. 